\title{
CENTER OF MASS AND $G$-LOCAL TRIVIALITY OF $G$-BUNDLES
}

\author{
KARSTEN GROVE
}

\begin{abstract}
Riemannian geometry techniques are used to give a short and constructive proof that a differentiable $G$-fibre bundle with compact fibre is $G$-locally trivial when $G$ is a compact Lie Group.
\end{abstract}

Introduction. The general notion of "center of mass" was introduced in [3] in order to give a constructive proof for the fact that any two $C^{1}$-close group actions of a compact Lie group $G$ on a compact differentiable manifold $M$ are equivalent. The information obtained from this constructive proof was important in proving an equivariant sphere-theorem (compare [4] and [5]). In addition, the "center of mass" was used in [4] and [5] to show that for any "almost homomorphism" between compact Lie groups there is a homomorphism close to it.

The purpose of the present note is to apply the "center of mass" to prove that any differentiable $G$-bundle with compact fibre is $G$-locally trivial when $G$ is a compact Lie group. This was already proved in Bierstone [1]. However, besides being simpler, our proof has the advantage of being constructive. It is therefore likely to be useful in specific problems in, e.g., Riemannian geometry (compare [4] and [5]).

It is our hope that these applications (among others) will make it clear that the "center of mass" is useful as a technical tool in differential geometry as well as in differential topology. It can be considered as a nonlinear approach to standard linear averaging methods. [6]).

1. The center of mass. We recall some facts from [3] (see also H. Karcher

Let $(X, \mu)$ be a measure space with $\mu(X)=1$ and let $M$ be a Riemannian manifold. A measurable map $f: X \rightarrow M$ is said to be almost constant if its image $f(X)$ is contained in a sufficiently small convex ball $B_{\rho}$ of radius $\rho$ (depending on the curvature; see [3]). $B_{\rho}$ is "sufficiently" small if the vectorfield

$$
\Upsilon(q)=\int_{X} \exp _{q}^{-1} \circ f d \mu
$$

where $\exp _{q}: T_{q} M \rightarrow M$ is the exponential map, has a unique zero in $B_{\rho}$. This point is called the center of mass of $f$ and it is denoted by $\mathcal{Q}(f)$ (it is also characterized as the unique minimum for the convex function

Received by the editors January 23, 1975.

AMS (MOS) subject classifications (1970). Primary 57E15; Secondary 53C20, 58D15.

Key words and phrases. $G$-bundle, $G$-triviality, center of mass, almost constant map, curvature. 


$$
J(q)=\int_{X} d(f(\cdot), q) d \mu \text { on } B_{\rho},
$$

where $d: M \times M \rightarrow \mathbf{R}$ is the distance function on $M$ ).

The important properties of $\mathcal{Q}(f)$ are:

(1.1) if $\varphi: X \rightarrow X$ is measure preserving, then $\mathcal{C}(f \circ \varphi)=\mathcal{C}(f)$;

(1.2) if $A: M \rightarrow M$ is an isometry, then $\mathrm{C}(A \circ f)=A(\mathcal{C}(f))$.

Both properties are easy consequences of the construction of $\mathcal{Q}(f)$.

Let now $X$ be a compact topological space with borel measure $\mu$. The Banach manifold $C^{0}(X, M)$ consisting of all continuous maps from $X$ to $M$ contains $M$ as a submanifold (all the constant maps from $X$ to $M$ ). In this case we can consider the set of "almost constant maps" $\mathfrak{A}$ as a tubular neighborhood of $M$ in $C^{0}(X, M)$ and $\mathcal{C}: \mathfrak{A} \rightarrow M$ as a differentiable retraction of $\mathfrak{A}$ onto $M$ (compare [3]).

2. $G$-bundles. $A$ differentiable $G$-fibre bundle consists of a differentiable fibre bundle $\pi: E \rightarrow B$ together with a Lie group $G$ acting differentiably on $E$ and $B$ such that $\pi$ is equivariant. Two such $G$-bundles $\pi: E \rightarrow B$ and $\pi^{\prime}: E^{\prime}$ $\rightarrow B^{\prime}$ are $G$-isomorphic if there is an equivariant bundle isomorphism between $\pi$ and $\pi^{\prime}$. Let $G_{b}=\{g \in G \mid g \cdot b=b\}$ denote the isotropy group of $G$ at $b \in B$. Then $G_{b}$ operates on the fibre $\pi^{-1}(b) . \pi: E \rightarrow B$ is said to be $G$-locally trivial if each point $b \in B$ has a $G_{b}$-invariant neighborhood $U_{b}$ such that $\pi_{l}: \pi^{-1}\left(U_{b}\right) \rightarrow U_{b}$ is $G_{b}$-isomorphic to the trivial $G_{b}$-bundle $U_{b} \times \pi^{-1}(b)$ $\rightarrow U_{b}$.

THEOREM 2.1. Let $\pi: E \rightarrow B$ be a differentiable $G$-fibre bundle with compact fibre. If $G$ is compact then $\pi: E \rightarrow B$ is $G$-locally trivial.

Proof. Since $G$ is compact we can choose Riemannian metrics on $E$ and on $B$ such that $G$ acts by isometries. Let $b_{0} \in B$ and put $\pi^{-1}\left(b_{0}\right)=F$. Note that when we endow $F$ with the induced metric from $E$ then $G_{b_{0}}$ operates on $F$ by isometries. Obviously we may as well assume that $G_{b_{0}}=G$ and that $E=B \times F$. Denote this given action on $E=B \times F$ by $\mu_{1}: G \times B \times F$ $\rightarrow B \times F$ and the product action of $G$ on $B$ and on $F$ by $\mu_{2}: G \times B \times F$ $\rightarrow B \times F$. We denote the actions of $G$ on $B$ and on $F$ by $\mu_{B}$ and $\mu_{F}$, respectively. Put $\Phi=P_{F} \circ \mu_{1}: G \times B \times F \rightarrow B \times F$, where $P_{F}: B \times F \rightarrow F$ is the projection onto $F$. Then $\Phi$ satisfies

$$
\begin{aligned}
\mu_{F} & =\Phi \mid: G \times\left\{b_{0}\right\} \times F \rightarrow F, \\
\Phi\left(g_{1} \cdot g_{2}, b, f\right) & =\Phi\left(g_{1}, \mu_{B}\left(g_{2}, b\right), \Phi\left(g_{2}, b, f\right)\right) .
\end{aligned}
$$

Let now

$$
\eta: G \times B \times F \rightarrow F ; \quad(g, b, f) \rightarrow \Phi\left(g^{-1}, b_{0}, \Phi(g, b, f)\right),
$$

and correspondingly,

$$
\hat{\eta}: B \times F \rightarrow C^{0}(G, F) ; \quad(b, f) \rightarrow \eta(\cdot, b, f) .
$$

We can choose a $G$-invariant neighborhood of $b_{0}$, say $U$ such that $\hat{\eta}(b)$ is "almost constant" for all $b \in U$. (Here we endow $G$ with bi-invariant metric 
and corresponding measure of total volume 1.) Put $\psi=e \circ \hat{\eta}_{l}: U \times F \rightarrow F$ and define $\Psi: U \times F \rightarrow U \times F$ by $\Psi(b, f)=(b, \psi(b, f))$ for all $(b, f) \in U$ $\times F$. Then

$$
\begin{aligned}
\mu_{2}(g, \Psi(b, f)) & =\left(\mu_{B}(g, b), \mu_{F}(g, \psi(b, f))\right) \\
& =\left(\mu_{B}(g, b), \Phi\left(g, b_{0}, \bigodot(\hat{\eta}(b, f))\right)\right) \quad \text { by }(2.2
\end{aligned}
$$

and

$$
\begin{aligned}
\Psi\left(\mu_{1}(g,(b, f))\right) & =\Psi\left(\mu_{B}(g, b), \Phi(g, b, f)\right) \\
& =\left(\mu_{B}(g, b), \mathcal{\bigodot}\left(\hat{\eta}\left(\mu_{B}(g, b), \Phi(g, b, f)\right)\right)\right)
\end{aligned}
$$

Now

$$
\begin{aligned}
\Phi\left(g, b_{0}, \mathcal{C}(\hat{\eta}(, f))\right) & =\bigodot\left(\Phi\left(g, b_{0}, \cdot\right) \circ \hat{\eta}(b, f)\right) \\
& =\bigodot\left(h \rightarrow \Phi\left(g h^{-1}, b_{0}, \Phi(h, b, f)\right)\right) \\
& =\bigodot\left(h \rightarrow \Phi\left(h^{-1}, b_{0}, \Phi(h g, b, f)\right)\right) \\
& =\bigodot\left(\hat{\eta}\left(\mu_{B}(g, b), \Phi(g, b, f)\right)\right)
\end{aligned}
$$

i.e.

$$
\mu_{2}(g, \Psi(b, f))=\Psi\left(\mu_{1}(g,(b, f))\right) \text { for all }(g, b, f) \in G \times U \times F .
$$

Since $\psi(b, \cdot): F \rightarrow F$ is $C^{1}$-close to $1_{F}$ when $b$ is close to $b_{0}$, we can find a neighborhood $V \subset U$ of $b_{0}$ in $B$ such that $\Psi: V \times F \rightarrow V \times F$ is a $G$-bundle isomorphism.

REMARK. Theorem 2.1 generalizes the result of Palais [7] and Calabi [2] about differentiable families of $G$-actions. Note also that the equivariant part of the pinching-theorem of [4], [5] can be formulated as follows. Let $M$ be a complete Riemannian manifold whose sectional curvature satisfies $0<\delta$ $\leqq K \leqq 1$ and let $G$ be a compact Lie group which operates on $M$ by isometries. There exists $a \delta_{0}<1$ (independent of $G$ and independent of $\operatorname{dim} M$ ) such that the G-bundle $E=T M \oplus M \times \mathbf{R} \rightarrow M$ is G-globally trivial when $\delta>\delta_{0}$.

\section{REFERENCES}

1. E. Bierstone, The equivariant covering homotopy property for differentiable G-fibre bundles, J. Differential Geometry 8 (1973), 615-622.

2. E. Calabi, On differentiable actions of compact Lie groups on compact manifolds, Proc. Conf. on Transformation Groups (New Orleans, La., 1967), Springer, New York, 1968, pp. 210-213. MR 39 \# 6347.

3. K. Grove and H. Karcher, How to conjugate $C^{l}$-close group actions, Math. Z. 132 (1973), 11-20.

4. K. Grove, H. Karcher and E. Ruh, Group actions and curvature, Invent. Math. 23 (1974), $31-48$

5. - - Jacobi fields and Finsler metrics on compact Lie groups with an application to differentiable pinching problems, Math. Ann. 211 (1974), 7-21.

6. H. Karcher, Jacobifeld-Techniken in der Riemannschen Geometrie, Lecture Notes in Math., Springer, Berlin and New York (to appear).

7. R. S. Palais, Equivalence of nearby differentiable actions of a compact group, Bull. Amer. Math. Soc. 67 (1961), 362-364. MR 24 \# A185. 